IJMMS 32:9 (2002) 565-572

PII. S0161171202013340

http://ijmms.hindawi.com

(c) Hindawi Publishing Corp.

\title{
ACCELERATION OF NONHYPERBOLIC SEQUENCES OF MANN
}

\author{
MÁRIO M. GRAÇA
}

Received 22 May 2001 and in revised form 2 November 2001

Mann's sequences are difficult to accelerate in the presence of a nonhyperbolic fixed point. New accelerators are constructed for Mann's sequences which are useful even for other sets of very slowly convergent sequences.

2000 Mathematics Subject Classification: 65B99, 65H05.

1. Introduction. One method for the numerical computation of a solution of a nonlinear equation of the form $x=g(x)$ is to choose an initial value $x_{0}$ and the iterative process $x_{n+1}=g\left(x_{n}\right)$ in the hope that the sequence $\left(x_{n}\right)$, generated by $g$, converges to a fixed point $x^{*}$ of $g$ (which is a solution of $g(x)-x=0$ ). To assure that such a sequence converges to $x^{*}$, we often use fixed-point theoretical results such as the Banach fixed-point theorem (see [15] for a survey on fixed point theorems). Namely, if an operator $A: X \mapsto X$, defined in a complete metric space $X$ is a $q$-contractor, that is, $d(A x, A y) \leq q d(x, y)$ with $0 \leq q<1$ and $d$ the metric in $X$, then the equation $x=A x$ has a unique solution $x^{*}$ in $X$ and for any $x_{0}$ in $X$, the iterative process $x_{n+1}=A x_{n}$ converges to $x^{*}$. So, for a real differentiable iteration function $g$ to be a $q$-contractor, we must have $\left|g^{\prime}\left(x^{*}\right)\right| \leq q<1$. When $g^{\prime}\left(x^{*}\right)=1$, the situation is somewhat different, and that is why this case has its own name-the nonhyperbolic case. Even when the existence and unicity of a fixed point for a nonlinear equation is guaranteed still remains the computational task of computing fast such a fixed point $x^{*}$ when $x^{*}$ is nonhyperbolic. The main aim of this work is precisely the computation of a fixed point $x^{*}$ (which is assumed to exist) for certain type of convergent fixed point sequences, $x_{n+1}=g\left(x_{n}\right)$ verifying $g^{\prime}\left(x^{*}\right)=1$, so converging very slowly. The type of sequences we will consider is generated by iteration functions $g$ of the form $g(x)=x+\phi(x)(f(x)-x)$, where $f:[0,1] \mapsto[0,1]$ has at least one fixed point $x^{*}, f^{\prime}\left(x^{*}\right)=1$ and $\phi\left(x^{*}\right) \neq 0$ (see Definition 2.1 for the specific type of sequences to be considered throughout the paper). This kind of sequences belongs to a larger class known as Mann's sequences [11]. Convergence results for (hyperbolic) Mann's sequences have been obtained by Dotson [4]; however, convergence issues of Mann's sequences are out of the scope of this work. We will always assume that the sequences in study are convergent, and we mainly address the question of how to accelerate their convergence.

Several authors tried to accelerate the convergence of sequences generated by iteration functions of the form $g(x)=x+\phi(x)(f(x)-x)$. For instance, Kowalewski in [9] showed that when $x^{*}$ is hyperbolic for $f\left(\left|f^{\prime}\left(x^{*}\right)\right|<1\right)$, the Aitken's process [1] and Ney's transformation [12] accelerate the convergence. However, she failed 
to find an accelerator in the nonhyperbolic case, that is, when $f^{\prime}\left(x^{*}\right)=1$. Nonhyperbolic Mann's sequences are logarithmic convergent fixed-point sequences, that is, sequences $\left(x_{n}\right)_{n} x^{*}$ such that $\lim _{n \rightarrow \infty}\left(x_{n+1}-x^{*}\right) /\left(x_{n}-x^{*}\right)=1$ (properties characterizing some general subsets of logarithmic real sequences are given in [8]).

Nonhyperbolic Mann's sequences are used here as an application of a general approach developed by Graça in [5, 6]. This approach is based on the following scheme: given an iteration map $g$, having an unknown nonhyperbolic fixed point $x^{*}$ constructs, in a first step, a new map $h$, with the same fixed point such that $h^{\prime}\left(x^{*}\right) \neq 1$ (for instance by a simple newtonisation of the function $x-g(x))$. In a second step, the function $h$ is used to construct another iteration map $H$ having the same fixed point but verifying $H^{\prime}\left(x^{*}\right)=0$. This means that a nonhyperbolic fixed point for $g$ becomes a super attracting fixed point for $H$. In other words, given a very slow convergent sequence generated by an iterative process governed by the map $g$, we can construct $H$ in order to generate a sequence converging rapidly to the same fixed point of $g$.

The construction process of the maps $h$ and $H$ is quite general and obeys to the single notion of combined iteration function (see Definition 3.1) introduced in Graça [5]. Combined iteration functions are useful in many other applications for the computation of nonhyperbolic fixed points. For instance, an obvious application is the computation of multiple roots of a polynomial without the explicit knowledge of their multiplicities.

The first two sections are devoted to the introduction of the necessary notions and definitions as well as to the proof that we can always accelerate the convergence of certain sets of nonhyperbolic sequences of Mann. The paper ends with numerical examples showing the acceleration effectiveness of our iteration functions $H$. In these examples, iterative processes, having at least order two of convergence, are obtained enabling the computation of good approximations of nonhyperbolic fixed points of Mann's sequences in a few iterations.

2. Definitions. In Dotson [4], a (normal) sequence of Mann $\left(x_{n}\right)$ is defined by

$$
x_{n+1}=x_{n}+\alpha_{n}\left(f\left(x_{n}\right)-x_{n}\right)=g\left(x_{n}, f\left(x_{n}\right), \alpha_{n}\right),
$$

where $f:[0,1] \rightarrow[0,1]$ is a Lipschitz function with a finite number of fixed points, and $\alpha_{n}$ is a real sequence such that

(a) $0<\alpha_{n}<1$, for all $n$,

(b) $\lim _{n \rightarrow \infty} \alpha_{n}=0$,

(c) $\sum_{n=0}^{\infty} \alpha_{n}$ divergent.

By definition, a sequence of Mann is a fixed-point sequence generated by an iteration function $g$, depending on a given function $f$ and an auxiliary sequence $\alpha_{n}$. This type of sequences bears Mann's name since in [11], Mann has studied a Cesàro process, choosing for $\left(\alpha_{n}\right)$ the sequence $(1 / n)$. For examples of Mann's sequences and conditions for the existence of a fixed point as well as the convergence of a normal sequence of Mann, see Dotson [4]. Our main interest here is not the study of Mann's sequences in general but rather the fast computation of nonhyperbolic fixed points of a certain type of sequences of Mann. 
Kowalewski [9] considered the question of accelerating the convergence of Mann's sequences, where $\alpha_{n+1}=\alpha_{n}\left(1-\alpha_{n}^{p}\right)$ and $p>1$. In particular, Kowalewski has shown that for a hyperbolic fixed point $x^{*}$ of $f$, either Ney's transformation or the Aitken's $\Delta^{2}$ process accelerates the convergence of $\left(x_{n}\right)$. The same work also presented some study cases for a nonhyperbolic fixed point of $f$ being, however, unable to find any accelerator. As it will be shown, we are always able to accelerate these nonhyperbolic fixed-point sequences by means of our iteration function $H$ (see examples in Section 4).

In this work, whenever we refer to a (nonhyperbolic) Mann's sequence, we are adopting the following definition.

DEFinition 2.1. Let $x^{*}$ be an isolated nonhyperbolic fixed point $\left(f^{\prime}\left(x^{*}\right)=1\right)$ of a given real function $f:[0,1] \mapsto[0,1]$, continuously differentiable in a neighbourhood $\mathscr{D}$ of $x^{*}$, and consider the sequence $x_{n+1}=g\left(x_{n}\right), x_{0} \in \mathscr{D}$, where

$$
g(x)=x+\phi(x)(f(x)-x)
$$

and $\phi:] 0,1[\rightarrow \mathbb{R}$ is

$$
\phi(x)=x\left(1-x^{p}\right), \quad p \in \mathbb{R}, p>1 .
$$

The sequence $\left(x_{n}\right)$ is called a nonhyperbolic sequence of Mann.

Note that for $\phi$ defined by (2.3) the sequence $\alpha_{n+1}=\phi\left(\alpha_{n}\right)$ is a fixed-point sequence verifying the conditions (a), (b), and (c) in Section 2. Indeed it is not hard to see that $\left(\alpha_{n}\right)$ is a decreasing sequence of positive terms converging to zero, and as $\alpha_{n+1}=$ $\alpha_{0} \prod_{i=0}^{n}\left(1-\alpha_{i}^{p}\right)$, we can easily deduce that $\sum_{i=0}^{\infty} \alpha_{i}^{p}$ diverges and also that the condition (c) is verified.

As we will see later, the sequence $\left(\alpha_{n}\right)$ does not play any particular role in the application of our accelerators for nonhyperbolic Mann's sequences.

Interesting cases arise when the function $f$ in (2.2) is polynomial and $x^{*}$ is a multiple root of $f(x)-x=0$ of multiplicity $m, m \geq 2$, that is, (see [2, page 87]) when

$$
f(x)=x+\left(x-x^{*}\right)^{m} a(x), \quad a\left(x^{*}\right) \neq 0,
$$

and $a(x)$ is continuous at $x=x^{*}$ (see Section 4).

With respect to the notion of accelerator, we will adopt a convenient definition for the present context of iteration functions.

DEFINITION 2.2. Let $g$ and $u$ be two continuously differentiable iteration functions in a neighbourhood $\mathscr{D}$ of a common fixed point $x^{*}, x_{n}=g\left(x_{n-1}\right) \underset{n}{\longrightarrow} x^{*}$, and $t_{n}=$ $u\left(t_{n-1}\right) \underset{n}{\rightarrow} x^{*}, x_{0} \in \mathscr{D}$.

We say that $u$ is an accelerator of $g$ in $\mathscr{D}$ (or the sequence $\left(t_{n}\right)$ converges faster than $\left.\left(x_{n}\right)\right)$ if $u^{\prime}\left(x^{*}\right)=0$ and $g^{\prime}\left(x^{*}\right) \neq 0$.

Note that by the mean value theorem and for all $n>0$, we have

$$
\begin{array}{cl}
x_{n}-x^{*}=g^{\prime}\left(\xi_{n}\right)\left(x_{n-1}-x^{*}\right), & \xi_{n} \in\left(x_{n-1}, x^{*}\right) \text { or } \xi_{n} \in\left(x^{*}, x_{n-1}\right), \\
t_{n}-x^{*}=u^{\prime}\left(\tau_{n}\right)\left(t_{n-1}-x^{*}\right), & \tau_{n} \in\left(t_{n-1}, x^{*}\right) \text { or } \tau_{n} \in\left(x^{*}, t_{n-1}\right) .
\end{array}
$$


Thus

$$
\frac{t_{n}-x^{*}}{x_{n}-x^{*}}=\frac{u^{\prime}\left(\tau_{n}\right)\left(t_{n-1}-x^{*}\right)}{g^{\prime}\left(\xi_{n}\right)\left(x_{n-1}-x^{*}\right)} .
$$

By the assumptions of $g$ and $u$, there exists an integer $N$ such that

$$
\left|\frac{t_{n}-x^{*}}{x_{n}-x^{*}}\right|<\left|\frac{t_{n-1}-x^{*}}{x_{n-1}-x^{*}}\right|, \quad \forall n>N .
$$

Therefore, $\lim _{n \rightarrow \infty}\left(t_{n}-x^{*}\right) /\left(x_{n}-x^{*}\right)=0$, which is the usual condition for faster convergence (see [3, page 2]).

3. Accelerating Mann's sequences. In the presence of a nonhyperbolic fixed point $x^{*}$ of a given iteration function $g$, it is possible to construct an accelerator for $g$ using a suitable combination of $g$ with another iteration function $h$ for which $x^{*}$ is a hyperbolic fixed point $\left(h^{\prime}\left(x^{*}\right) \neq 1\right)$. For that purpose, in [5] we have defined a combined iteration function.

DEFINITION 3.1 (combined iteration function). Let $g$ and $h$ be any two differentiable functions in a neighborhood $\mathscr{D}$ of a common fixed point $x^{*}$ and $h^{\prime}\left(x^{*}\right) \neq 1$. A combined iteration function $H=C(g, h)$ is defined by

$$
H(x)=C(g, h)(x)=\frac{h(x)-g(x) h^{\prime}(x)}{1-h^{\prime}(x)}, \quad \forall x \in \mathscr{D} .
$$

The requirement of $x^{*}$ to be a common fixed point of $g$ and $h$ in the definition of $H$ implies that $x^{*}$ is also a fixed point of $H$.

A straightforward computation of $H^{\prime}(x)$ gives the following proposition.

Proposition 3.2. If $x^{*}$ is a nonhyperbolic fixed point of $g$ with $g^{\prime}\left(x^{*}\right)=1$, then $H^{\prime}\left(x^{*}\right)=0$ where $H=C(g, h)$.

Proposition 3.2 shows that the iteration map $H$ is always an accelerator of the given nonhyperbolic map $g$. The main question now is how to choose the map $h$ to be used in $C(g, h)$.

Next we show that, in general (if the given map $g$ has good differentiability properties), a natural choice for $h$ is Newton's map.

Proposition 3.3. Consider $x^{*}$ to be an isolated fixed point of a sufficiently many times differentiable iteration function $u$ such that

$$
u^{\prime}\left(x^{*}\right)=1, \quad u^{(j)}\left(x^{*}\right)=0, \quad 2 \leq j \leq m-1, \quad u^{(m)}\left(x^{*}\right) \neq 0,
$$

for some integer $m \geq 2$. Let $h$ be given by

$$
h(x)= \begin{cases}\frac{u(x)-x u^{\prime}(x)}{1-u^{\prime}(x)} & \text { if } x \neq x^{*}, \\ x^{*} & \text { if } x=x^{*}\end{cases}
$$


Then $h$ is differentiable at $x^{*}$ and

$$
0<h^{\prime}\left(x^{*}\right)=1-\frac{1}{m}<1 .
$$

Proof. The result follows using Taylor's series for $u$ and $u^{\prime}$ in the computation (by definition) of $h^{\prime}\left(x^{*}\right)$ (see, e.g., [7, 10, 13]).

The function $h$ of the previous proposition is a particular type of a combined iteration function; namely, $h(x)=C(x, u)$, which also coincides with the Newton's iteration function $N(x)=x-\Psi(x) / \Psi^{\prime}(x)$ for $\Psi(x)=x-u(x)$. Hereafter, we refer to $h$ given by (3.3) as the newtonisation of $u$.

An immediate consequence of Propositions 3.3 and 3.2 is the fast computation of a multiple root of an equation $x-u(x)=0$. Choose for $h$ the newtonisation of $u$; that is, $h(x)=C(x, u)$, and for $g$ either $u$ or even the identity map (i.e., $g(x)=x$ ), then $H=C(g, h)$ will be an accelerator of the original map. Note that if we choose for $h$ the Newtonisation of $u$ and for $g$ the identity map, then $H=C(g, h)$ corresponds to a double newtonisation of the original map $u$. Although under the conditions of Proposition 3.3 we get for $h$ a $(1-1 / m)$-contractor, this is not a necessary condition for the function $h$ to be used in Proposition 3.2. We can choose $h$ to be even a function verifying $\left|h^{\prime}\left(x^{*}\right)\right|>1$, the respective iterative process generated by $H=C(g, h)$ still being an accelerator of the nonhyperbolic process generated by $g$. So, combined iteration functions are also useful for the computation of nonhyperbolic fixed points even when we choose an iteration function $h$ such that the sequence $x_{n+1}=h\left(x_{n}\right)$ is locally divergent.

We apply the previous results to Mann's nonhyperbolic sequences.

THEOREM 3.4. Let $g(x)=x+\phi(x)(f(x)-x)$ be a Mann's iteration function as in Definition 2.1, and $m \geq 2$ an integer such that

$$
f\left(x^{*}\right)=x^{*}, \quad f^{\prime}\left(x^{*}\right)=1, \quad f^{(j)}\left(x^{*}\right)=0, \quad 2 \leq j \leq m-1, \quad f^{(m)}\left(x^{*}\right) \neq 0 .
$$

For $u$, either $f$ or $g$ and $h=C(x, u)$, then

(i) $h^{\prime}\left(x^{*}\right)=1-1 / m$;

(ii) $H=C(g, h)$ and $H=C(f, h)$ are accelerators of $g$.

PRoof. (i) The derivative of order $k$ of $g$ is

$$
g^{(k)}(x)=\sum_{i=0}^{k}\left(\begin{array}{l}
k \\
i
\end{array}\right) \phi^{(k-i)}(x)(f(x)-x)^{(i)},
$$

and so the hypotheses on $f$ imply that $g$ and $f$ satisfy the hypotheses of the function $u$ of the Proposition 3.3. So, $h=C(x, g)$ and $h=C(x, f)$ satisfy $h^{\prime}\left(x^{*}\right)=1-1 / m$.

(ii) By (i), when $h=C(x, f)$ or $h=C(x, g)$ we have $h^{\prime}\left(x^{*}\right) \neq 1$, and so the Proposition 3.2 gives that $H=C(g, h)$ and $H=C(f, h)$ are accelerators.

4. Numerical examples. Let $f(x)=-(1 / 3) x^{3}+(1 / 2) x^{2}+(3 / 4) x+1 / 24$ (see [9, page 138]), having the fixed point $x^{*}=0.5$ with $f^{\prime}\left(x^{*}\right)=1, f^{(2)}\left(x^{*}\right)=0$, and $f^{(3)}\left(x^{*}\right)=$ -2 (i.e., $x^{*}$ is a root of multiplicity $m=3$ for the equation $f(x)-x=0$ ). Consider 
TABLE 4.1. $h=C(x, f), H=C(g, h)$.

\begin{tabular}{lll}
\hline$g$ & $h$ & $H$ \\
\hline 0.8 & 0.8 & 0.8 \\
0.797408 & 0.7 & 0.505184 \\
0.794862 & 0.633333 & 0.5 \\
0.792361 & 0.588889 & 0.5 \\
\hline
\end{tabular}

the auxiliary iteration function $\phi(x)=x\left(1-x^{2}\right)$. The corresponding nonhyperbolic Mann's iteration function is

$$
\begin{gathered}
g(x)=x+\phi(x)(f(x)-x)=\frac{x}{24}\left(25-6 x+11 x^{2}-2 x^{3}-12 x^{4}+8 x^{5}\right), \\
g^{\prime}\left(x^{*}\right)=1, \quad g^{(2)}\left(x^{*}\right)=0, \quad g^{(3)}\left(x^{*}\right)=-0.75 \neq 0 .
\end{gathered}
$$

The hyperbolic iteration function $h=C(x, f)$ is given by

$$
h(x)=C(x, f)(x)=\frac{1}{6}(1+4 x)
$$

and $h^{\prime}\left(x^{*}\right)=1-1 / m=2 / 3 \neq 1$.

The combined iteration function $H=C(g, h)$ is

$$
H(x)=C(g, h)(x)=\frac{1}{12}\left(6-x+6 x^{2}-11 x^{3}+2 x^{4}+12 x^{5}-8 x^{6}\right),
$$

leading to an iterative process of order three of convergence [13, pages 9-12] since $H^{\prime}\left(x^{*}\right)=H^{(2)}\left(x^{*}\right)=0$, and $H^{(3)}\left(x^{*}\right)=1.5 \neq 0$.

Table 4.1 shows the values of the first three iterations, respectively, for the iteration functions $g, h$, and $H$, beginning with $x_{0}=0.8$ and computed using Mathematica [14] with machine precision.

Now, take the same $f$ and $p=100$ in the auxiliary iteration function $\phi$ given in (2.3). The corresponding nonhyperbolic Mann's iteration function $g$ is

$$
g(x)=x+\frac{1}{24} x(-1+2 x)^{3}\left(-1+x^{100}\right),
$$

with $g^{\prime}\left(x^{*}\right)=1, g^{(2)}\left(x^{*}\right)=0, g^{(3)}\left(x^{*}\right)=-1$.

Now, choose $h=C(x, g)$

$$
\begin{aligned}
h(x)=C(x, g)(x) & =\frac{6 x^{2}+100 x^{101}-206 x^{102}}{-1+8 x+101 x^{100}-208 x^{101}}, \\
h^{\prime}\left(x^{*}\right) & =0.666667 \neq 1 .
\end{aligned}
$$

The accelerator $H=C(g, h)$ has a cumbersome expression which will be omitted for the sake of simplicity. We get

$$
H^{\prime}\left(x^{*}\right)=0, \quad H^{(2)}\left(x^{*}\right)=-1.33333 \neq 0 .
$$


TABLE 4.2. $h=C(x, g), H=C(g, h)$.

\begin{tabular}{lll}
\hline$g$ & $h$ & $H$ \\
\hline 0.8 & 0.8 & 0.8 \\
0.7928 & 0.711111 & 0.496525 \\
0.786166 & 0.647077 & 0.499992 \\
0.780025 & 0.601504 & 0.499998 \\
\hline
\end{tabular}

So, the iteration function $H$ leads to a second-order iterative process (see Table 4.2). Note that there can be considerable loss of numerical accuracy if too many iterations are used.

5. Conclusions. In this paper, we have developed a new method of computing nonhyperbolic fixed points for sequences of Mann. The procedure is illustrated with two test sequences which are known to be difficult to accelerate.

The same ideas can be used to construct algorithms for the computation of nonhyperbolic fixed points of a general iteration function in $\mathbb{R}$.

ACKNOWLEDGMENTS. The author would like to thank the anonymous referees for their helpful suggestions. This work was supported by IDMEC-IST through the program POCTI of FCT (Portugal).

\section{REFERENCES}

[1] A. C. Aitken, On Bernoulli's numerical solution of algebraic equations, Proc. Roy. Soc. Edinburgh 46 (1926), 289-305.

[2] K. E. Atkinson, An Introduction to Numerical Analysis, 2nd ed., John Wiley \& Sons, New York, 1989.

[3] C. Brezinski and M. Redivo Zaglia, Extrapolation Methods: Theory and Practice, Studies in Computational Mathematics, vol. 2, North-Holland Publishing, Amsterdam, 1991.

[4] W. G. Dotson Jr., On the Mann iterative process, Trans. Amer. Math. Soc. 149 (1970), $65-73$.

[5] M. M. Graça, Nonhyperbolic fixed points and flat iteration functions, to appear in Experiment. Math.

[6] _ Simple accelerators for logarithmic fixed point sequences, Problems in Applied Mathematics and Computational Intelligence (N. Mastorakis, ed.), World Scientific and Engineering Society Press, New Jersey, 2001, pp. 82-86.

[7] E. Isaacson and H. B. Keller, Analysis of Numerical Methods, John Wiley \& Sons, New York, 1966.

[8] C. Kowalewski, Accélération de la convergence pour certaines suites à convergence logarithmique [Acceleration of convergence for certain sequences of logarithmic convergence], Padé Approximation and Its Applications, Amsterdam, 1980, Lecture Notes in Mathematics, vol. 888, Springer, Berlin, 1981, pp. 263-272 (French).

[9] _ Possibilites d'acceleration de la convergence logarithmique, Ph.D. thesis, Université de Lille, France, 1981.

[10] R. Kress, Numerical Analysis, Graduate Texts in Mathematics, vol. 181, Springer-Verlag, New York, 1998.

[11] W. R. Mann, Mean value methods in iteration, Proc. Amer. Math. Soc. 4 (1953), 506-510.

[12] A. Ney, Observations concernant la formule d'extrapolation d'Aitken, Rev. Anal. Numér. Théorie Approximation 5 (1976), no. 1, 59-62 (French). 
[13] J. F. Traub, Iterative Methods for the Solution of Equations, Prentice-Hall Series in Automatic Computation, Prentice-Hall, New Jersey, 1964.

[14] S. Wolfram, The Mathematica Book, 3rd ed., Cambridge University Press, Cambridge, 1996.

[15] E. Zeidler, Nonlinear Functional Analysis and Its Applications. I. Fixed-Point Theorems, Springer-Verlag, New York, 1986.

MÁrio M. GraÇA: InSTITUTO Superior TÉCNico, Departamento MATEMÁtica, Av. Rovisco PAIS, 1049-001 LisboA, PORTUGAL

E-mail address: mgraca@math.ist.ut 1.pt 


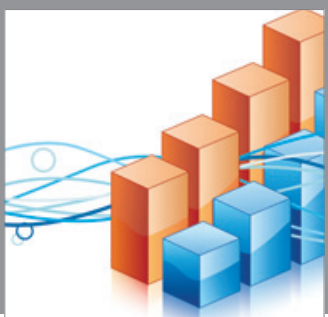

Advances in

Operations Research

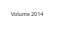

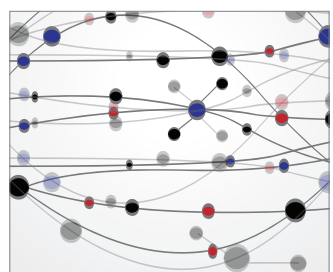

\section{The Scientific} World Journal
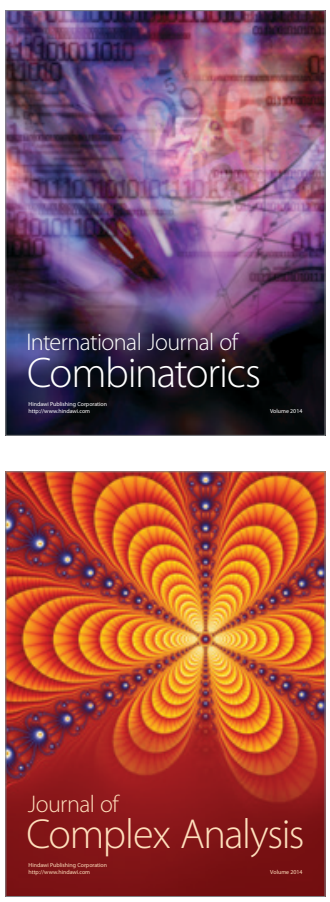

International Journal of

Mathematics and

Mathematical

Sciences
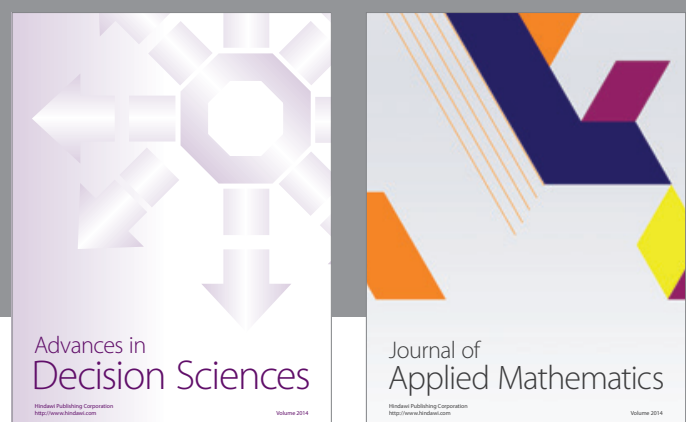

Journal of

Applied Mathematics
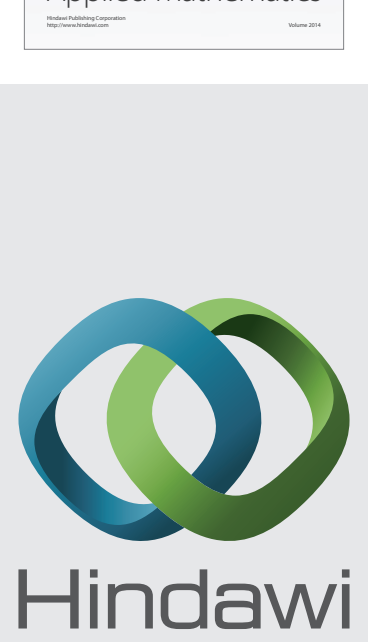

Submit your manuscripts at http://www.hindawi.com
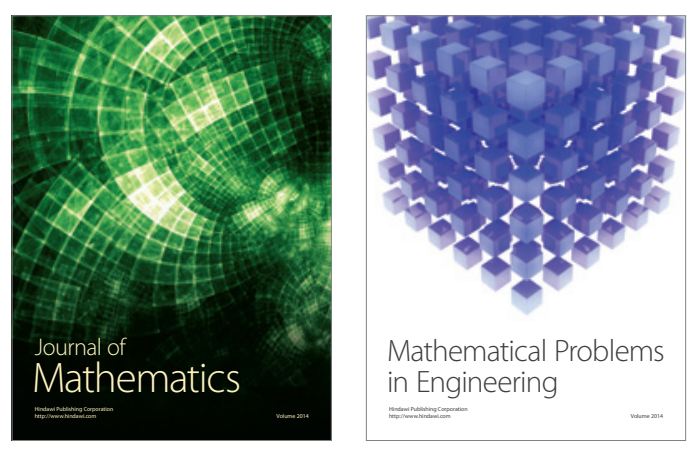

Mathematical Problems in Engineering
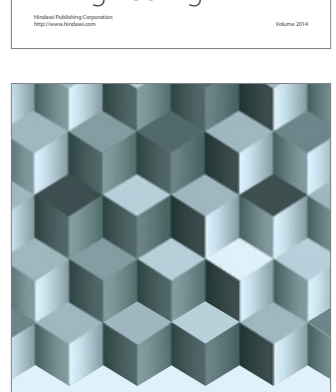

Journal of

Function Spaces
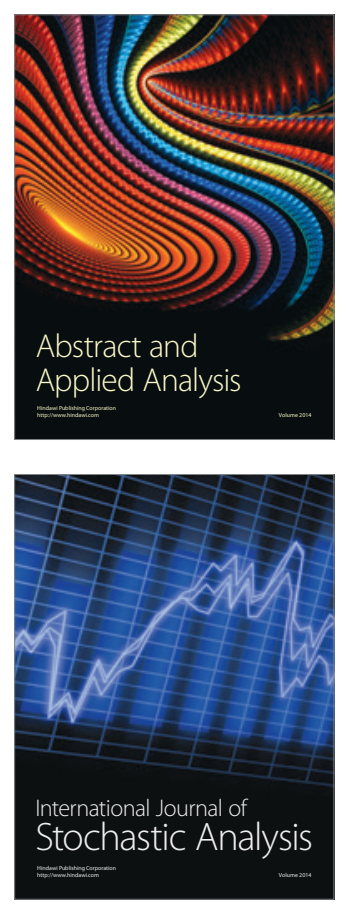

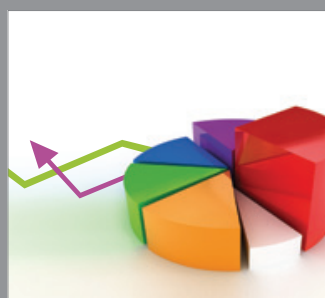

ournal of

Probability and Statistics

Promensencen
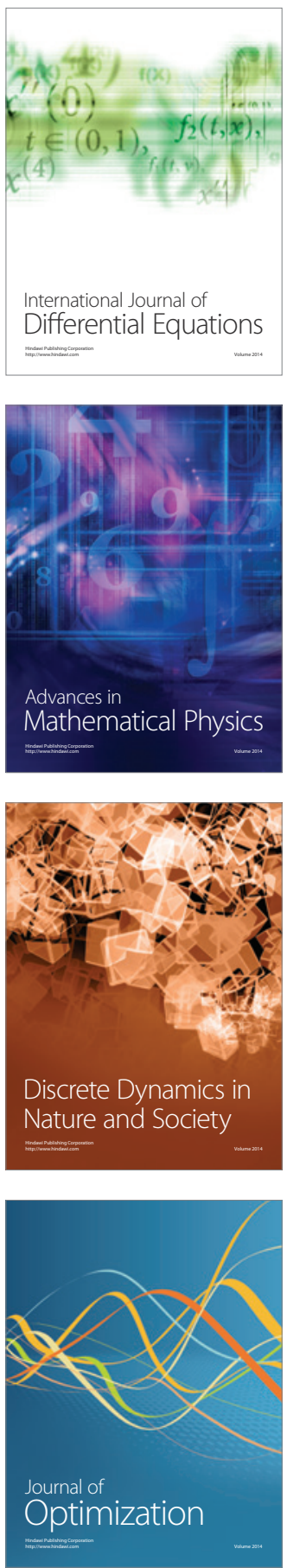